\title{
Comment on the paper "Microsystem Technologies (2018) 24:4965-4979"
}

\section{Asterios Pantokratoras ${ }^{1}$}

Received: 17 January 2020 / Accepted: 16 March 2020/Published online: 23 March 2020

(c) Springer-Verlag GmbH Germany, part of Springer Nature 2020

\section{Abstract}

In the above paper two serious errors have been found.

In the above paper the heat equation is as follows (equation 18 in Ezzat and El-Bary 2018)

$$
\begin{aligned}
& \left(1+\tau_{0} \frac{\partial^{v}}{\partial t^{v}}\right)\left(\rho C_{E} \frac{\partial^{2} T}{\partial t^{2}}+T_{0} \gamma \frac{\partial^{2} e}{\partial t^{2}}\right) \\
& =\left(a_{1} k+a_{2} \tau_{a} k^{*}\right) \frac{\partial^{3} T}{\partial t \partial x^{2}}+a_{3} k^{*} \frac{\partial^{2} T}{\partial x^{2}}+S \sigma_{0} \Pi \frac{\partial^{2} T}{\partial x^{2}}
\end{aligned}
$$

where $\tau_{0}, \tau_{a}(\mathrm{sec})$ are the relaxation times, $t(\mathrm{sec})$ is the usual time, $v$ is a dimensionless exponent, $a_{1}, a_{2}$ are dimensionless coefficients and $k\left(\right.$ Kelvin $\left.^{-1} \mathrm{~kg} \mathrm{~m} \mathrm{sec}{ }^{-3}\right)$ is the thermal conductivity. The number 1 is a pure number (dimensionless) whereas the units of the term $\tau_{0} \frac{\partial^{0}}{\partial t^{0}}$ are $\sec ^{1-v}$. In Physics it is not allowed to add quantities with different units and for that reason the term $\left(1+\tau_{0} \frac{\partial^{v}}{\partial t^{v}}\right)$ is wrong. This means that the Eq. (1) is also wrong.

Above equation (20) in Ezzat and El-Bary (2018) a series of dimensionless parameters have been defined and the dimensionless equation (21) is as follows

$$
\begin{aligned}
& C \frac{\partial^{3} \theta(x, t)}{\partial t \partial x^{2}}+A \frac{\partial^{2} \theta(x, t)}{\partial x^{2}} \\
& \quad=\left(1+\tau_{0} \frac{\partial^{v}}{\partial t^{v}}\right)\left(\frac{\partial^{2} \theta(x, t)}{\partial t^{2}}+\varepsilon \frac{\partial^{2} e(x, t)}{\partial t^{2}}\right)
\end{aligned}
$$

where the parameter $C$ is defined in the following equation

$$
C=a_{1} k+a_{2} \tau_{a} k^{*}
$$

The terms $a_{1} k$ and $a_{2} \tau_{a} k^{*}$ in Eq. (1) must have the same units. Therefore the units of $k^{*}$ must be Kelvin $^{-1} \mathrm{~kg} \mathrm{~m} \mathrm{sec}{ }^{-4}$ and the units of constant $\mathrm{C}$ are Kelvin $^{-1} \mathrm{~kg} \mathrm{~m} \mathrm{sec}^{-3}$.

In the dimensionless Eq. (2) the parameters $x, t, \theta, \varepsilon, e$ are dimensionless whereas the parameters $C, \tau_{0}$ are dimensional. In a dimensionless equation all terms must be dimensionless. For that reason the Eq. (2) is also wrong and the results in Ezzat and El-Bary (2018) are also wrong.

\section{Reference}

Ezzat MA, El-Bary AA (2018) Unified GN model of electrothermoelasticity theories with fractional order of heat transfer. Micosyst Technol 24:4965-4979

Publisher's Note Springer Nature remains neutral with regard to jurisdictional claims in published maps and institutional affiliations.

Asterios Pantokratoras apantokr@civil.duth.gr

1 School of Engineering, Democritus University of Thrace, 67100 Xanthi, Greece 